\title{
Human defensin 5 is stored in precursor form in normal Paneth cells and is expressed by some villous epithelial cells and by metaplastic Paneth cells in the colon in inflammatory bowel disease
}

\author{
R N Cunliffe, F R A J Rose, J Keyte, L Abberley, W C Chan, Y R Mahida
}

\begin{abstract}
Background and aims-Intestinal epithelial cell derived antimicrobial peptides of the defensin family may play a major role in host defence against microorganisms. Our aims were to (i) isolate, characterise, and investigate the processing of human defensin 5 (HD-5) in normal Paneth cells and (ii) investigate expression of HD-5 in active inflammatory bowel disease (IBD). Methods-Antiserum raised against chemically synthesised putative mature HD-5 was used for immunohistochemistry and purification of HD-5 from extracts of normal terminal ileal crypts.

Results-In normal and Crohn's disease terminal ileum, HD-5 immunoreactivity was seen in Paneth cells and in some villous epithelial cells. Normal colonic mucosa did not express HD-5 but HD-5 immunoreactivity was seen in cells in the colonic crypt region of many IBD samples. $N$-terminal amino acid sequence analysis of HD-5 purified from normal terminal ileal Paneth cells consistently showed the predicted sequence of the precursor form of the peptide. Following stimulation of isolated intact normal terminal ileal crypts, a truncated form of HD-5, with the $N$-terminal sequence GEDNQLAIS, was detected in the supernatant.

Conclusions-(i) HD-5 is present only in the precursor form in normal terminal ileal Paneth cells and is processed to the mature form during and/or after secretion, (ii) some villous epithelial cells express HD-5, and (iii) HD-5 is expressed by metaplastic Paneth cells in the colon in IBD.
\end{abstract}

(Gut 2001;48:176-185)

Keywords: inflammatory bowel disease; human defensin 5; Paneth cells

Department of Pharmaceutical Sciences, University of

Nottingham, UK

W C Chan

Correspondence to: Y R Mahida, Division of Gastroenterology, University Hospital, Queen's Medical Centre, Nottingham NG7 2UH, UK.

Yash.Mahida@nottingham. ac.uk

Accepted for publication 22 August 2000

The gastrointestinal tract is constantly exposed to a large population of microorganisms and their products. This exposure may occur following ingestion of contaminated food or beverages but is especially prominent in the colon where a large population of bacteria is resident in the lumen. The total population of approximately $10^{14}$ bacteria consists of about 400 different species. ${ }^{1}$ The mechanisms by which the host intestinal mucosa interacts with such a microbial load remain to be characterised. Recent studies in animal models suggest that dysregulation or impairment of host responses to the resident microorganisms may lead to chronic inflammatory bowel disease (IBD)..$^{3}$

Host-microbial interactions in the normal gastrointestinal tract may vary in different sites. In contrast with the normal colon (where the presence of the resident flora may be of benefit to the host), a significant bacterial population in the nutrient rich environment of the small intestinal lumen would be disadvantageous to the host. The number of bacteria resident in the small intestine falls markedly from $10^{3}-10^{9}$ in the ileum to $0-10^{5}$ in the upper small bowel. ${ }^{1}$ In addition to gastric acid and motility, it is likely that mucosal factors are responsible for maintaining the relative sterility of the small intestine. Such mucosal factors may represent components of the adaptive and innate immunity, ${ }^{4}$ and in which epithelial cells play a major role. In recent years, epithelial cell mediated innate host defences in the form of antimicrobial peptides and proteins have generated considerable interest. ${ }^{5}$ While mature enterocytes are capable of expressing antimicrobial peptides and proteins, ${ }^{6-8}$ Paneth cells have been the main focus of interest in this field. ${ }^{9}$ In addition to the antimicrobial proteins lysozyme, ${ }^{10}$ and secretory phospholipase $\mathrm{A}_{2}{ }^{11}{ }^{12}$ studies in mice have shown that Paneth cells express antimicrobial peptides of the $\alpha$-defensin family, which have been designated cryptdins. ${ }^{9}{ }^{13-16}$ In addition to contribution to innate immunity in the crypt lumen, cryptdins may also influence the function of neighbouring cells. ${ }^{17}$ Murine Paneth cells have been shown to express numerous isoforms of cryptdin. ${ }^{15}$ In contrast, complementary cDNAs of only two $\alpha$-defensins, human defensins 5 and 6 (HD-5 and HD-6), have been shown to be expressed by Paneth cells in humans. ${ }^{18}{ }^{19}$ While HD-5 has been localised to Paneth cells and a recombinant version of the peptide has been
Abbreviations used in this paper: IBD, inflammatory bowel disease; HD-5, HD-6, human defensins 5 and 6; RP-HPLC, reverse phase-high performance liquid chromatography; TFA, trifluoroacetic acid; PBS, phosphate buffered saline; ITF, intestinal trefoil factor; HBSS, Hanks balanced salt solution; LPS, lipopolysaccharide; SDS-PAGE, sodium dodecyl sulphate-polyacrylamide gel electrophoresis; PVDF, polyvinylidene difluoride; TTBS, Tris-Tween buffered saline; CFU, colony forming unit. 
produced, ${ }^{20}{ }^{21}$ the native peptide has yet to be fully characterised.

In this study, we have raised antisera against chemically synthesised HD-5 and demonstrated expression of HD-5 in Paneth cells and also some epithelial cells in the villous region of the small intestine. Expression of HD-5 was absent in the normal colonic mucosa but strong immunoreactivity was seen in some epithelial cells in sections of mucosa with active ulcerative colitis and Crohn's colitis. Using the antisera, native HD-5 was isolated and purified from acid extracts of normal terminal ileal crypts by cation exchange chromatography and reverse phase-high performance liquid chromatography (RP-HPLC). N-terminal sequence analysis consistently showed that HD-5 is present only in its precursor form in normal terminal ileal Paneth cells. Our studies following stimulation of isolated crypts with lipopolysaccharide and carbamylcholine chloride suggest that processing of pro-HD-5 to the mature form occurs during and/or after its secretion by Paneth cells.

\section{Materials and methods}

CHEMICAL SYNTHESIS OF HD-5 AND PRODUCTION OF ANTI-HD5 ANTISERA

The putative mature form of HD5 (amino acid residues 63-94; fig 1) was chemically synthesised by an automatic peptide synthesiser (Model 431A, Applied Biosystems, Foster City, California, USA) using Fmoc chemistry. It was purified by RP-HPLC using a $10 \times 100$ mm C18 preparative column (Brownlee column, Applied Biosytems) with a solvent gradient of acetonitrile in $0.1 \%$ trifluoroacetic acid (TFA) from $3.5 \%$ to $21 \%$ over the first 10 minutes, then from $21 \%$ to $42 \%$ over the next 20 minutes, and from $42 \%$ to $70 \%$ over the final minute. The purified synthetic peptide was characterised by $N$-terminal amino acid sequence analysis and mass spectrometry and was subsequently used to raise antisera. For the primary immunisation, New Zealand White rabbits were injected (subcutaneously) with $330 \mu \mathrm{g}$ of the synthetic peptide (in $500 \mu \mathrm{l}$ of normal saline and $500 \mu \mathrm{l}$ of complete Freund's adjuvant). Two boosts were given at 30 day intervals in the same way, except that incomplete, rather than complete, Freund's adjuvant was used. Blood was drawn prior to immunisation (preimmune sample) and 10 days after each boost and the separated serum was stored at $-80^{\circ} \mathrm{C}$ in the presence of $0.02 \%$ sodium azide.

TISSUE SAMPLES

Fresh normal and IBD terminal ileal and colonic mucosal samples were obtained from intestinal operation specimens resected for carcinoma (the normal mucosal samples were obtained more than $5 \mathrm{~cm}$ from the tumour) or active IBD. Additional normal and active IBD mucosal tissue was obtained from the pathology archive at University Hospital, Nottingham.
IMMUNOHISTOCHEMISTRY

Five micron sections of formalin fixed paraffin embedded intestinal tissue samples (normal duodenum $n=9$, normal jejunum $n=2$, normal terminal ileum $n=12$, normal colon $n=6$, terminal ileal Crohn's disease $n=2$, colonic (rectal or proximal colonic) Crohn's disease $n=8$, ulcerative colitis (involving rectum or proximal colon) $\mathrm{n}=13$ ) were dewaxed in xylene and rehydrated through graded alcohols. Antigen retrieval was achieved by incubation with Pronase (Sigma, St Louis, Missouri, USA) at $1 \mathrm{mg} / \mathrm{ml}$ for 20 minutes at room temperature. Immunoperoxidase staining was performed using a Vectastain Elite Universal ABC kit (Vector Laboratories, Burlingame, California, USA) according to the manufacturer's instructions. In brief, sections were incubated with blocking serum at room temperature for 30 minutes and then incubated for one hour at room temperature with a 1:2000 dilution of rabbit anti-HD-5 antiserum in phosphate buffered saline (PBS). Rabbit preimmune serum diluted 1:2000 in PBS was used as a negative control. Endogenous peroxidase activity was quenched by incubation in $0.3 \%$ $\mathrm{H}_{2} \mathrm{O}_{2}$ in methanol for 20 minutes. Bound antibodies were detected by incubation with a biotinylated antirabbit IgG, followed by an avidin-biotinylated horseradish peroxidase complex. Peroxidase activity was developed with diaminobenzidine tetrahydrochloride and the sections were counterstained with haematoxylin. Immunoperoxidase staining was similarly performed on selected sections using anti-lysozyme (1:100; NeoMarkers, Union City, California, USA) and anti-intestinal trefoil factor (ITF; $1: 400^{22}$ ) polyclonal antisera diluted in PBS. In addition, to verify specificity of the immunostaining with the anti-HD-5 antiserum, immunoadsorption was performed by incubating synthetic HD- 5 at a concentration of $20 \mu \mathrm{g} / \mathrm{ml}$ in diluted anti-HD-5 antiserum for three hours at room temperature. In subsequent studies on tissue sections, immunolabelling with immunoadsorbed anti-HD-5 antiserum was compared with untreated antiserum.

ISOLATION OF SMALL INTESTINAL CRYPTS

Fresh normal terminal ileal mucosal samples were obtained from operation specimens resected for caecal carcinoma and mucosal strips were dissected from the submucosa and weighed (generally $2-4 \mathrm{~g}$ total). Intact crypts and crypt epithelial cells were detached from the basement membrane using EDTA, as previously described. ${ }^{23}$ In brief, mucosal strips were incubated in $1 \mathrm{mM}$ dithiothreitol (Sigma) for 15 minutes at room temperature. Epithelial cells were subsequently detached by three 30 minute incubations in $1 \mathrm{mM}$ EDTA (Sigma) at $37^{\circ} \mathrm{C}$ with continuous gentle stirring. Between each incubation mucosal samples were washed with calcium and magnesium free Hanks balanced salt solution (HBSS; Gibco BRL, Gaithersburg, Maryland, USA). Crypt epithelial cells, largely in the form of intact crypt structures, were obtained after the second and third incubations in EDTA, leaving an intact lamina propria covered by basement membrane. 
In some experiments investigating secretion of HD-5, isolated crypts were washed three times in HBSS supplemented with calcium (Gibco BRL) and incubated at $37^{\circ} \mathrm{C}$ with $5 \%$ $\mathrm{CO}_{2}$ for one hour in RPMI (Gibco BRL) containing $10^{-5} \mathrm{M}$ carbamylcholine chloride (carbachol; Sigma) or $10 \mu \mathrm{g} / \mathrm{ml}$ lipopolysaccharide (LPS; from Escherichia coli 055:B5; Sigma). Crypt culture supernatants were dialysed against distilled water for 18 hours with a $1 \mathrm{kDa}$ cut off dialysis membrane (Spectra Por, Pierce and Warriner, Chester, UK ) at $4^{\circ} \mathrm{C}$ in the presence of peptidase inhibitors $(1 \mathrm{mM}$ phenylmethylsulphonyl fluoride, $1 \mathrm{mg} / \mathrm{ml} \mathrm{pep-}$ statin, and $1 \mathrm{mg} / \mathrm{ml}$ leupeptin; Sigma), lyophilised, and resuspended in $0.1 \%$ acetic acid for further analysis.

PREPARATION OF CRYPT CELL EXTRACTS

The isolated epithelial crypts obtained during the second and third EDTA incubation steps were pooled and washed three times in HBSS by centrifugation at $400 \mathrm{~g}$ for 10 minutes. They were resuspended in $10 \%$ acetic acid, subjected to one cycle of rapid freeze-thawing to bring about cell lysis, and stirred for 18 hours at $4^{\circ} \mathrm{C}$ in the presence of peptidase inhibitors (as above). Supernatants (containing cationic peptides) were clarified by centrifugation (at $400 \mathrm{~g}$ for 10 minutes), lyophilised, resuspended in $0.1 \%$ acetic acid, and stored at $-80^{\circ} \mathrm{C}$ for further use.

ISOLATION OF HD-5 FROM CRYPT CELL EXTRACTS AND CULTURE SUPERNATANTS

Acid extracts of epithelial crypts isolated from terminal ileal mucosal samples of five different resection specimens were pooled, applied to a cation exchange column (SP Sepharose Fast Flow; Pharmacia Biotech, Uppsala, Sweden) and eluted using a $0-1 \mathrm{M} \mathrm{NaCl}$ gradient. Eluted fractions were dialysed against distilled water for 18 hours with a $1 \mathrm{kDa}$ cut off dialysis membrane (Spectra Por, Pierce and Warriner, Chester, UK), lyophilised, and resuspended in $0.1 \%$ acetic acid. Fractions that were immunoreactive for HD- 5 were identified by dot blot and subsequently western blot analyses. Positive fractions were further purified by RPHPLC using a $2.1 \times 30 \mathrm{~mm} \mathrm{C18}$ analytical column (Brownlee column; Applied Biosystems) with a linear gradient of acetonitrile in $0.1 \%$ TFA from $3.5 \%$ to $70 \%$ over 37 minutes. All major RP-HPLC peak fractions were analysed by AU-PAGE and western blot analyses. Selected fractions were also analysed by

$\begin{array}{ll}\frac{\text { Signal }}{\text { HD-5 (1-19) }} & \text { MRTIAILAAILLVALQAQA } \\ \frac{\text { Propiece }}{\text { HD-5 (20-62) }} & \text { ESLQERADEATTQKQSGEDNQDLAISFAGNGLSALRTSGSQAR } \\ \frac{\text { Mature peptide }}{\text { HD-5 (63-94) }} & \text { ATCYCRTGRCATRESLSGVCEISGRLYRLCCR }\end{array}$

Figure 1 Predicted amino acid sequence of human defensin 5 (HD-5), based on the ${ }_{c D N A}{ }^{18}$ Single letter amino acid code for prepro HD-5 is shown. The N-terminus of mature HD-5 is speculative. Conserved cysteines, characteristic of the a-defensin family, are shown in bold and amino acid residue numbering is shown in parentheses.
Tris-Tricine $16.5 \%$-sodium dodecyl sulphatepolyacrylamide gel electrophoresis (SDSPAGE). Purified HD-5 in RP-HPLC fractions was analysed by $N$-terminal amino acid sequencing. In addition, following transfer from AU-PAGE to polyvinylidene difluoride (PVDF) membranes, $N$-terminal sequence analysis of individual bands of protein was performed. Similar studies were also performed on extracts of crypts isolated from two other resection specimens.

Culture supernatants of isolated and stimulated intact crypts were analysed by AU-PAGE, Tris-Tricine SDS-PAGE, and western blot, and further purified by RP-HPLC as above. HD-5 was identified in RP-HPLC fractions by dot blot and subsequently analysed by $\mathrm{N}$-terminal amino acid sequencing.

GEL ELECTROPHORESIS

Crypt cell extracts were analysed at various stages during the HD-5 purification procedure by AU-PAGE, as previously described ${ }^{6}$. In brief, acid urea $(6.25 \mathrm{M})-15 \%$ polyacrylamide minigels were prerun with $5 \%$ acetic acid for one hour at $150 \mathrm{~V}$. Samples were electrophoresed with $5 \%$ acetic acid at $150 \mathrm{~V}$ until the methyl green dye front had reached the end of the gel, which was then stained with Coomassie blue. Tris-Tricine SDS-PAGE Ready Gels (Bio-Rad Laboratories, Hemel Hempstead, UK) were also used, according to the manufacturer's instructions.

IMMUNOBLOTTING

The presence of immunoreactive HD-5 in crypt cell extracts, purified extract fractions, and crypt culture supernatants was determined by dot blot and western blot analyses using the anti-HD-5 antisera. Synthetic HD-5 and human neutrophil peptide-1 (human defensin 1; Bachem Feinchemikalien AG, Switzerland) were used as standards. For dot blot, $10 \mu \mathrm{l}$ volumes of sample in $0.1 \%$ acetic acid were applied to PVDF membranes (Pro Blot membranes, Applied Biosystems) using a microfiltration apparatus attached to a water-vac system. For western blot analysis, peptides were electroblotted from AU-PAGE gels to PVDF membranes in $0.7 \%$ acetic acid $/ 10 \%$ methanol at $180 \mathrm{~mA}$ for 10 minutes using a Transblot cell (Bio-Rad). Membranes were fixed in $0.05 \%$ glutaraldehyde in Tris-Tween buffered saline (TTBS) for one hour, blocked in $2.5 \%$ skimmed milk in TTBS for 30 minutes, and incubated with a 1:2000 dilution of the HD-5 antisera in $2.5 \%$ skimmed milk/TTBS for one hour at room temperature. Where necessary, a 1:2000 dilution of rabbit preimmune antisera was used as a negative control. Blots were washed three times in TTBS and bound antibodies were subsequently visualised using the Vectastain Elite ABC kit as described above.

PROTEIN SEQUENCING AND MASS SPECTROMETRY $\mathrm{N}$-terminal amino acid sequencing of both synthetic HD-5 and that isolated and purified from crypt cells and culture supernatants was performed by Edman degradation chemistry using 
an Applied Biosystems 473A automated sequencer, as previously described. ${ }^{6}$ Electrospray mass spectrometry analysis of purified peptides was performed using a Micromass Platform machine (Micromass UK Ltd, Wythenshaw, $\mathrm{UK}$ ) and for the sample containing HD-5 purified from isolated (but not stimulated/cultured) ileal crypts, the raw data were transformed using the MaxEnt 1 algorithm.

ANTIMICROBIAL ASSAY

Antimicrobial activity of HD-5 isolated and purified as described above was studied using a colony forming unit (CFU) assay. A phoP mutant (CS015) of Salmonella typhimurium (gift from Dr S Miller, Massachusetts General Hospital, Boston, Massachusetts, USA), which is sensitive to defensins, ${ }^{24}$ was used as the test organism. An overnight culture of bacteria in $3 \%$ trypticase soy broth was subcultured at $1: 100$ and grown for three hours to mid logarithmic phase at $37^{\circ} \mathrm{C}$. Bacterial density was estimated photometrically, based on the fact that an $\mathrm{OD}_{620}$ of 0.2 is equal to $5 \times 10^{7}$ $\mathrm{CFU} / \mathrm{ml}$, and a working dilution of approximately $10^{6} \mathrm{CFU} / \mathrm{ml}$ was prepared in $10 \mathrm{mM}$ sodium phosphate buffer ( $\mathrm{pH} 7.4)-1 \%$ trypticase soy broth. A sample of $10 \mu \mathrm{l}$ of a $200 \mu \mathrm{g} / \mathrm{ml}$ solution of isolated HD-5 in $0.1 \%$ acetic acid was mixed with $90 \mu \mathrm{l}$ of bacterial suspension;
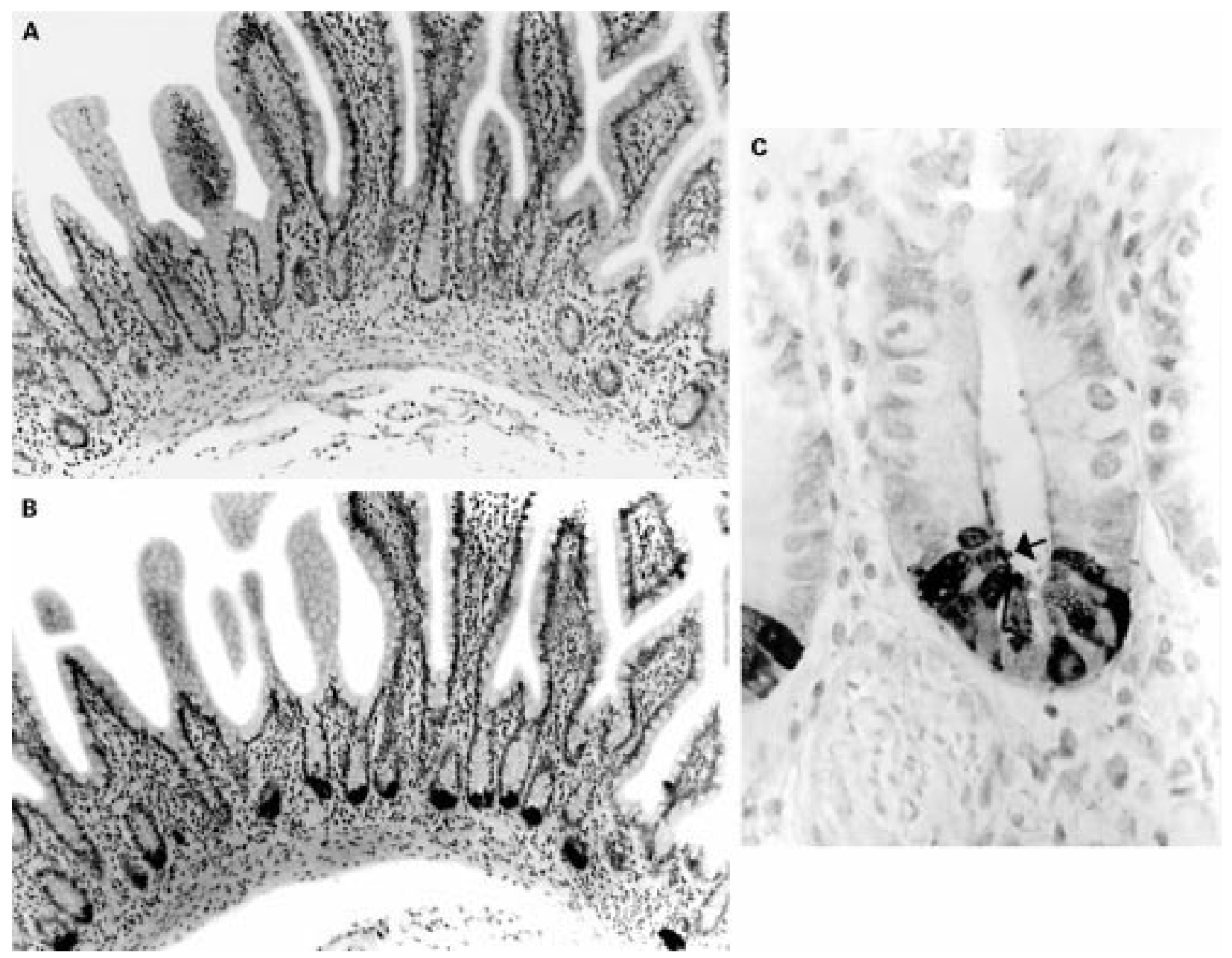

\section{Results}

PRODUCTION OF SYNTHETIC HD-5

$N$-terminal amino acid sequence analysis of the purified synthetic mature HD-5 obtained revealed the expected $N$-terminus (fig 1 ; first 10 $N$-terminal amino acid residues analysed). The purified synthetic peptide was homogeneous by RP-HPLC (not shown) and mass spectrometry revealed a mass of $3582 \mathrm{Da}$. This corresponds exactly with the expected mass of the fully oxidised putative mature form of HD-5 in which all cysteines have formed disulphide bonds. This

Figure 2 Normal terminal ileal mucosa stained with preimmune serum (A) and anti-human defensin 5 antisera (B, $C)$. The antisera reacts strongly with Paneth cells, and immunoreactive material can be seen in the crypt lumen ( $C$, arrow). 
suggests that the synthetic peptide had spontaneously oxidised during the purification process.

IMMUNOHISTOCHEMISTRY

The anti-HD-5 antisera showed strong immunoreactivity to Paneth cells in the normal small intestine (duodenum, jejunum, and terminal ileum), often with immunoreactive material in the crypt lumen, suggesting active granule secretion (fig 2A-C). In addition, in five of 12 normal terminal ileal and one of two jejunal mucosal samples studied (but none of the duodenal samples), occasional epithelial cells located in the villus region and towards the top of crypts were noted to be immunoreactive for HD-5 in a fine granular pattern, often confined to the apical half of the cell (figs $3 \mathrm{~A} ; 4 \mathrm{~A}, \mathrm{C}, \mathrm{D}$ ). These cells were morphologically distinct from Paneth cells. Similar HD-5 containing villous epithelial cells were also noted in both of the terminal ileal Crohn's disease samples studied. Specificity of HD-5 immunoreactivity for villous epithelial cells and Paneth cells was demonstrated by complete loss of immunoreactivity when the anti-HD-5 antiserum was preadsorbed with synthetic HD-5 (fig 3B). Studies using sequential sections showed that the HD-5 expressing villous epithelial cells express ITF (fig 4A, B) but not lysozyme (fig 4C, D, E, F). In the normal colon, no HD-5 immunoreactive cells were seen (fig 5A). However, scattered cells with strong HD-5 immunoreactivity were seen in four of eight colonic Crohn's disease and eight of 13 ulcerative colitis mucosal samples (fig $5 \mathrm{~B}$, C). These HD-5 positive cells were often seen in the crypt region. Studies using sequential sections revealed that these cells were morphologically identical to Paneth cells on haematoxylin and eosin staining, and also that they contained lysozyme on immunoperoxidase staining (not shown).

\section{PURIFICATION AND CHARACTERISATION OF}

NATIVE HD-5

Anti-HD-5 antiserum was used to facilitate the isolation and purification of HD-5 present in Paneth cells of the normal small intestine. Crypts were isolated from normal terminal ileal mucosal samples obtained from five different resection specimens, extracted with acetic acid, pooled, and applied to a cation exchange column. Fractions eluting between $0.4 \mathrm{M}$ and $1 \mathrm{M} \mathrm{NaCl}$ were immunoreactive for HD-5 by dot blot analysis (fig 6). Immunoblot analyses after acid-urea PAGE showed that HD-5 in all of these eluted fractions demonstrated similar electrophoretic mobility. Fractions eluting between $0.8 \mathrm{M}$ and $1 \mathrm{M} \mathrm{NaCl}$ demonstrated the strongest HD-5 immunoreactivity. Among these, fractions containing the most protein, as visualised on Coomassie blue stained AUPAGE, were pooled (as shown in the bracketed region of fig 6) and used for further purification by RP-HPLC.

On RP-HPLC, several eluted peaks were collected and one of these (indicated by an asterisk $\left(^{\star}\right)$ in fig $7 \mathrm{~A}$ and shown as a single RP-HPLC peak in fig 7B) was immunoreactive for HD-5. $N$-terminal amino acid sequencing of this peak revealed the predicted $\mathrm{N}$-terminal

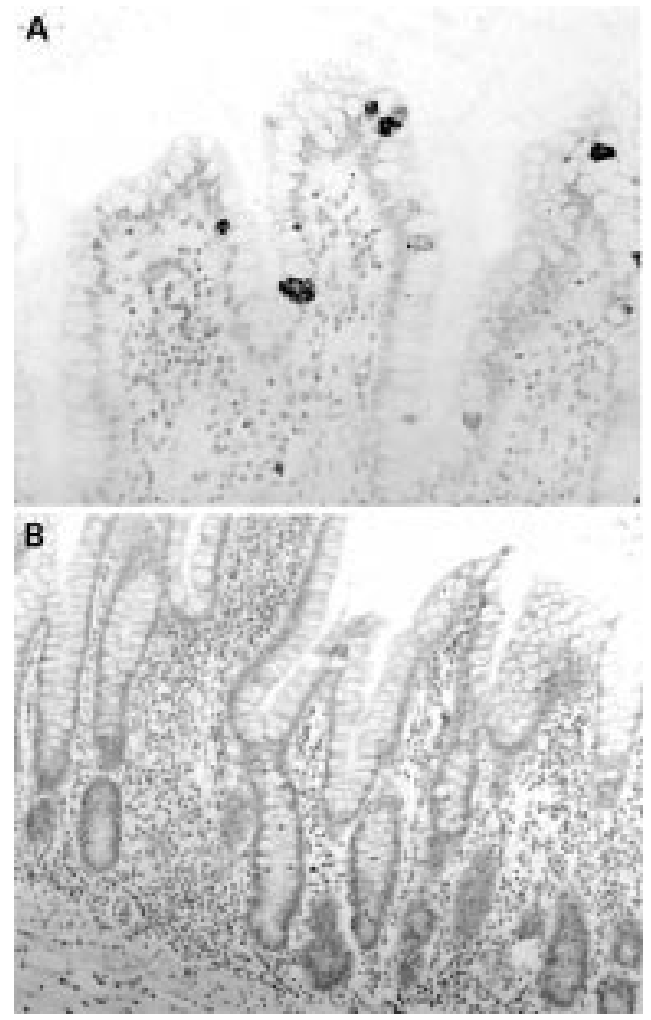

Figure 3 Normal terminal ileal mucosa (A) stained with anti-human defensin 5 (HD-5) antiserum showing occasional villous epithelial cells strongly immunoreactive for HD-5. (B) Section from the same specimen as (A) and stained with anti-HD-5 antiserum that had been preadsorbed with synthetic HD-5. Positive staining of HD-5 containing Paneth cells and villous epithelial cells is completely abolished.

sequence of the precursor form of $\mathrm{HD}-5$ (amino acids 20-94; figs 1, 7B). Mass spectral analysis of purified HD-5 (same sample as used for RP-HPLC in fig 7B) demonstrated the presence of a single major component with a mass of $8103.76 \mathrm{Da}$ (fig 7C). The expected mass of the precursor form of HD-5, deduced from the amino acid sequence (amino acids 20-94; fig 1) is 8103.83 Da. AU-PAGE (fig 8A) and Tris-tricine SDS-PAGE analyses of this single peak showed one discrete protein band and a doublet/broad band with slower migration. Protein bands on the AU-PAGE gel were transferred to a PVDF membrane and used for $N$-terminal amino acid sequence analyses (fig 8A). All three amino acid sequences obtained were similar and identical to the predicted $\mathrm{N}$-terminal sequence of the precursor form of HD-5. Studies on crypt extracts obtained from two other resection specimens also produced similar results.

The purified precursor form of HD- 5 did not demonstrate antimicrobial activity against the phoP mutant of $S$ typhimurium, which is sensitive to defensins. This is shown by a $2 \log$ increase in CFU after incubation of purified precursor HD-5 with the phoP mutant of $S$ typhimurium (average $\mathrm{CFU} / \mathrm{ml}$ at 0 hours, $1.2 \times 10^{6}$; after three hours, $2.0 \times 10^{8}$ ), and is similar to the result obtained using $0.1 \%$ acetic acid as a negative control (average $\mathrm{CFU} / \mathrm{ml}$ at 0 hours, $1.1 \times 10^{7}$; after three hours, $1.5 \times 10^{8}$ ). In contrast, the antimicrobial peptide magainin, 
A
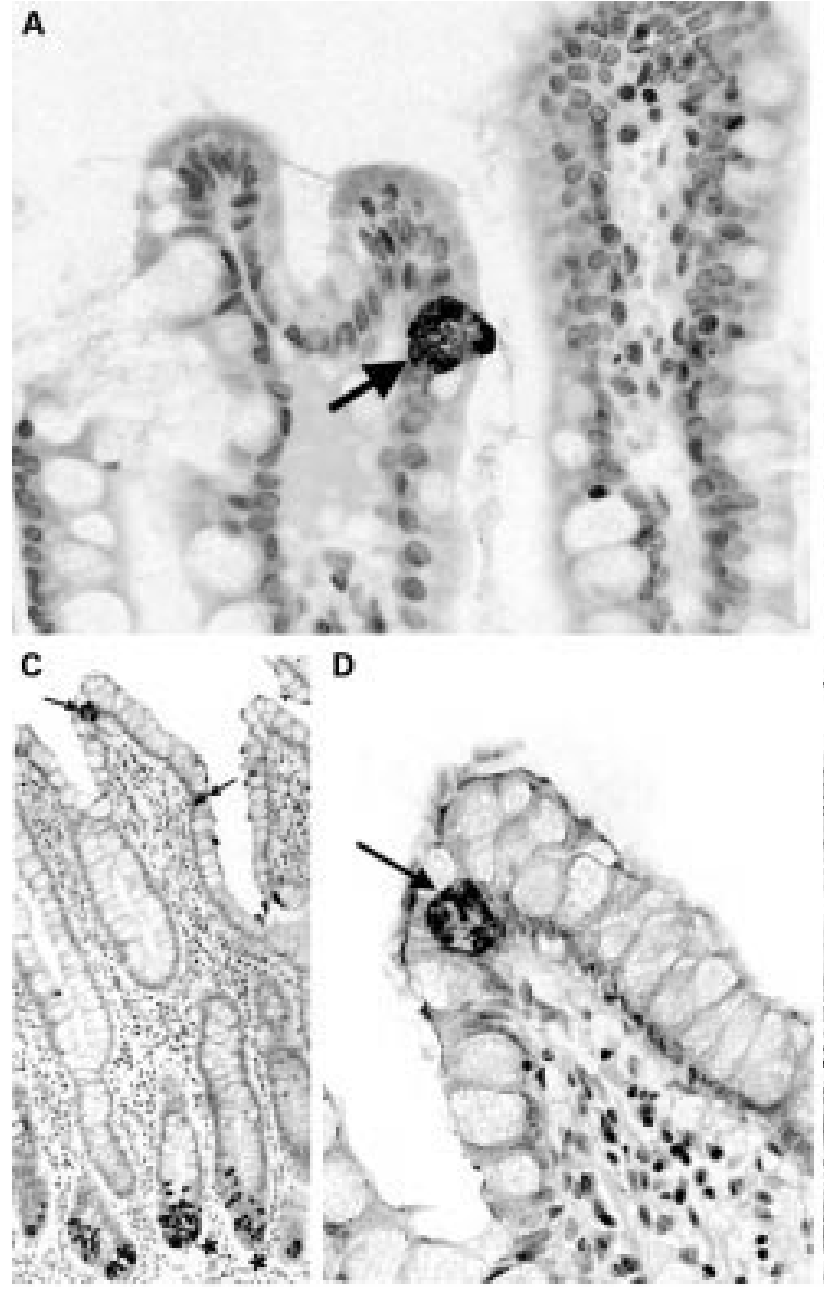

D
B

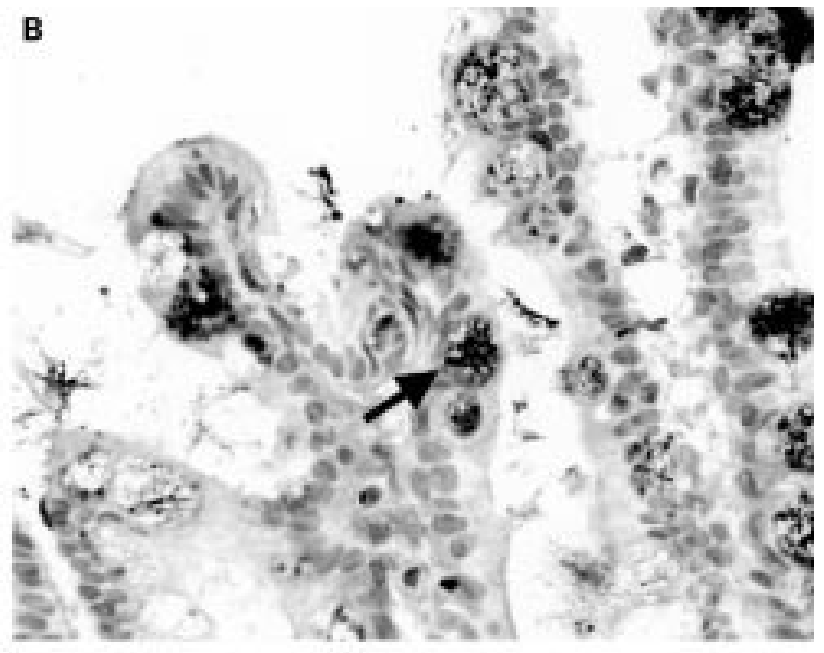

$\mathbf{F}$

Figure 4 Sequential sections of normal terminal ileal mucosa stained with anti-human defensin 5 (HD-5) antiserum (A) and anti-intestinal trefoil factor (ITF) antiserum (B). The arrowed HD-5 immunoreactive villous epithelial cell also expresses ITF. A similar pair of sequential sections is stained with anti-HD-5 antiserum $(C, D)$ and anti-lysozyme antiserum $(E, F)$. As can be seen in the low power views $(C, E)$, ileal Paneth cells express both HD-5 and lysozyme. (D, F) High power views of the same sections showing that the HD-5 immunoreactive villous epithelial cells (arrows) do not express lysozyme.

which was used as a positive control, demonstrated potent antimicrobial activity (average $\mathrm{CFU} / \mathrm{ml}$ at 0 hours, 0 ; after three hours, $\left.1.0 \times 10^{3}\right)$.

HD-5 SECRETION BY ISOLATED PANETH CELLS As HD-5 is present in the Paneth cells in the precursor form only, studies were performed to determine whether it is processed during secretion from the cells. Secretion by Paneth cells in isolated and intact epithelial crypts was stimulated using bacterial LPS and carbamylcholine chloride. Supernatant samples were subjected to AU-PAGE and Tris-tricine SDSPAGE, followed by immunoblot analyses. In addition to a band corresponding to the precursor form of HD-5, an additional HD-5 immunoreactive band of lower molecular weight was also seen (fig $8 \mathrm{~B}$ ). Such a pattern was consistently seen in separate western blot analyses of supernatant samples of stimulated crypts isolated from five separate ileal resection specimens (that is, from five subjects). Culture supernatant of stimulated crypts isolated from one resection specimen was applied to RPHPLC and $N$-terminal amino acid sequencing of the purified HD-5 immunoreactive fraction revealed an $\mathrm{N}$-terminally truncated version of the precursor form of HD-5 ( $N$-terminal sequence: GEDNQLAIS, amino acids 36-94; figs $1,8 \mathrm{~B})$. Further studies in which HD-5 was purified (by RP-HPLC) from pooled culture supernatant samples of stimulated crypts obtained from four other resection specimens showed the same $N$-terminally truncated version of the precursor form of HD-5 (Nterminal sequence: GEDNQLAIS).

\section{Discussion}

We have chemically synthesised the predicted mature form of HD- 5 based on the cDNA sequence $^{18}$ and used it to raise antisera. The subsequent immunohistochemical studies on sections of normal small intestinal mucosa showed strong immunoreactivity in Paneth cells, which was abolished when the antisera was preadsorbed with synthetic HD-5 (in addition, no immunostaining was demonstrated when the preimmune antisera was used). The anti-HD-5 antibody was subsequently used to facilitate the isolation and purification of native HD-5 from acid extracts of epithelial crypts isolated from fresh terminal ileal mucosal samples. During these and other (unpublished) studies, we did not see cross reactivity of the anti-HD-5 antisera 

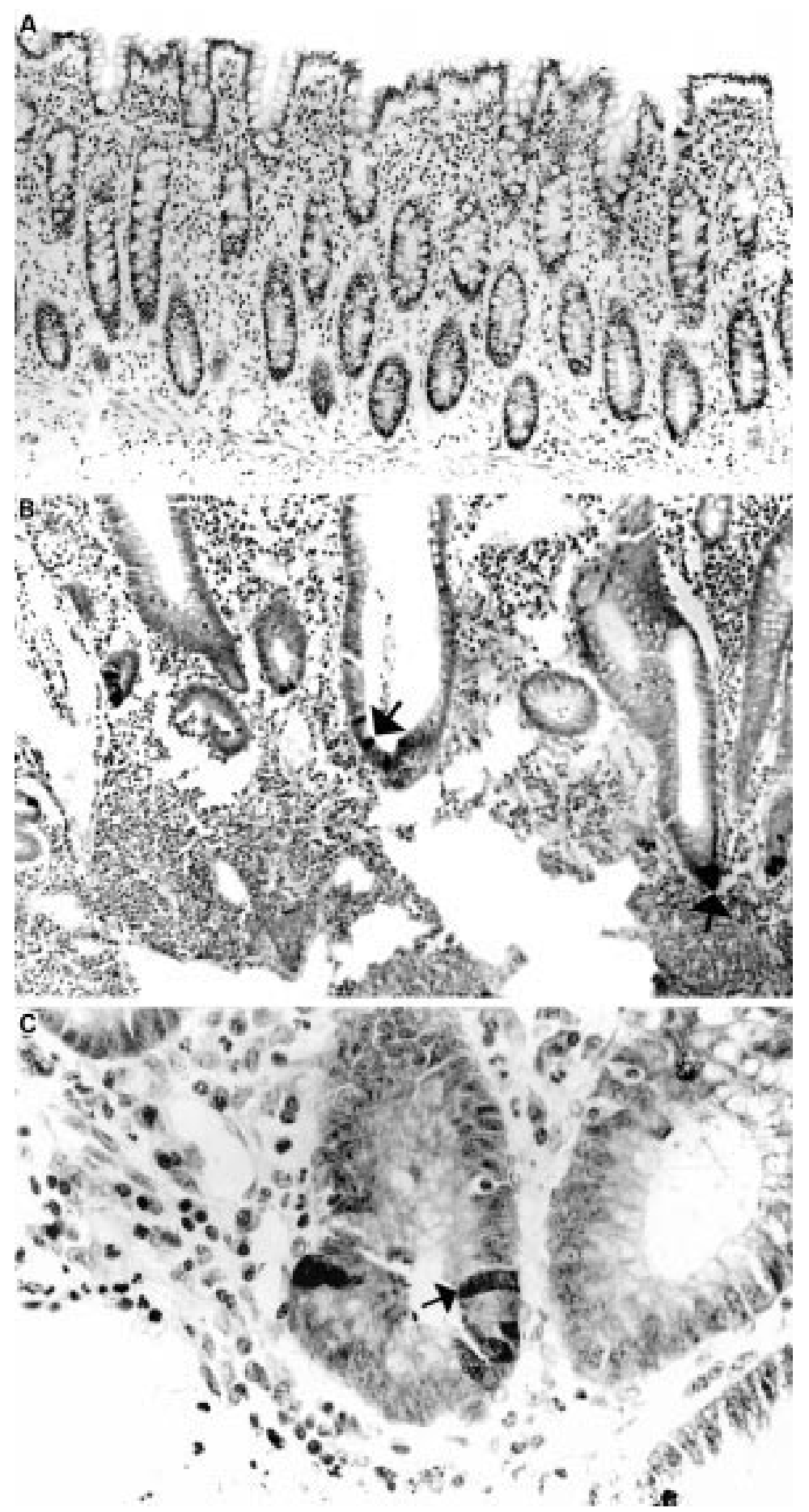

Figure 5 Sections of normal $(A)$ and ulcerative colitis $(B, C)$ colonic mucosal sections stained with anti-human defensin 5 (HD-5) antiserum. No HD-5 positive cells are present in (A). HD-5 immunoreactive epithelial cells (some of which are arrowed) are seen in the crypt regions of the sections with active ulcerative colitis $(B, C)$.

to any other entity. Therefore, these studies suggest that the antisera is specific for HD-5.

Our immunohistochemical studies showed that in addition to Paneth cells, some villous epithelial cells in a number of terminal ileal mucosal samples also expressed HD-5. Similar HD-5 immunoreactive villous epithelial cells were also seen in sections of terminal ileal Crohn's disease. These cells appear morphologically distinct from Paneth cells and do not express lysozyme (a Paneth cell specific prod-

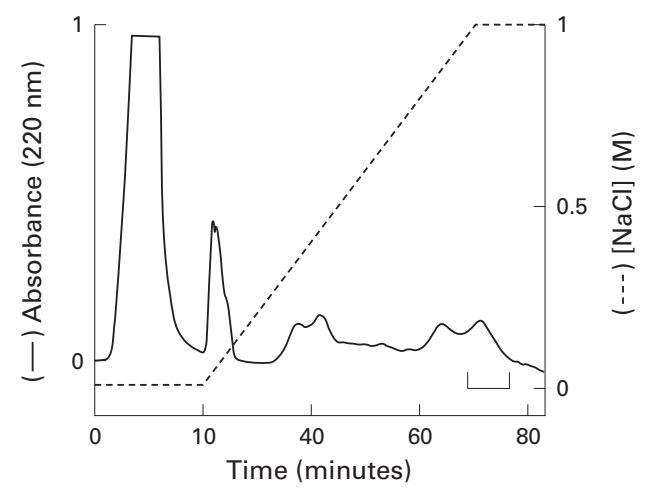

Figure 6 Cation exchange chromatography of ileal crypt cell extract. Acid extracts of terminal ileal crypt cells (obtained from five different resection specimens) were pooled and applied to a cation exchange column and eluted fractions containing human defensin 5 (HD-5) were identified by immunoblotting. Fractions eluting between 0.8 and $1 \mathrm{M} \mathrm{NaCl}$ demonstrated the strongest $H D-5$ immunoreactivity. Among these, fractions shown in the bracketed region were pooled for further purification by $R P$-HPLC.

uct) but express the goblet cell specific product ITF. $^{22}$ The nature and identity of these cells is unclear but they may represent the previously reported and rare "intermediate cells" which contain Paneth cell-like granules and mucin but not lysozyme. ${ }^{25} 26$ The function of these cells is unknown but their expression of HD-5 suggests that they may have a role in protection of the small intestinal mucosa from microbial invasion.

No HD-5 positive cells were seen in sections of normal colonic mucosa. However, HD-5 immunoreactive cells were present in the crypt region of a large proportion of colonic samples with active ulcerative colitis or Crohn's disease. Studies on sequential sections suggest that the HD-5 expressing cells in the crypt region are metaplastic Paneth cells, which also express lysozyme (Mason and Taylor, ${ }^{10}$ Geller and Thung, ${ }^{27}$ and our unpublished observations). We have also demonstrated expression of HD-5 mRNA in colonic epithelial cells of mucosal samples with IBD (unpublished observations). It is likely that induction of HD-5 expression in the colon with active IBD represents the host epithelial response to luminal bacteria and their products. Recently, we have also shown expression of polymorphonuclear defensins in the surface epithelial cells (but not metaplastic Paneth cells) in active IBD. ${ }^{8}$ In other recent studies, expression of HD-5 in the female genital tract has also been shown to be upregulated by inflammation. ${ }^{28}$ Moreover, expression of epithelial $\beta$-defensins may also be induced by inflammation or infection. ${ }^{29}$

To determine the amino acid sequence of the native peptide stored in normal small intestinal Paneth cells, HD-5 was isolated and purified from acid extracts of isolated epithelial crypts. Native HD-5 eluted over a wide gradient of $\mathrm{NaCl}$ but western blot analysis showed HD-5 immunoreactive bands of similar electrophoretic mobility in all the eluted fractions. RP-HPLC analysis of HD-5 purified from isolated (but not cultured/stimulated) ileal crypts demonstrated the presence of a single peak. Mass spectral analysis of this sample showed 

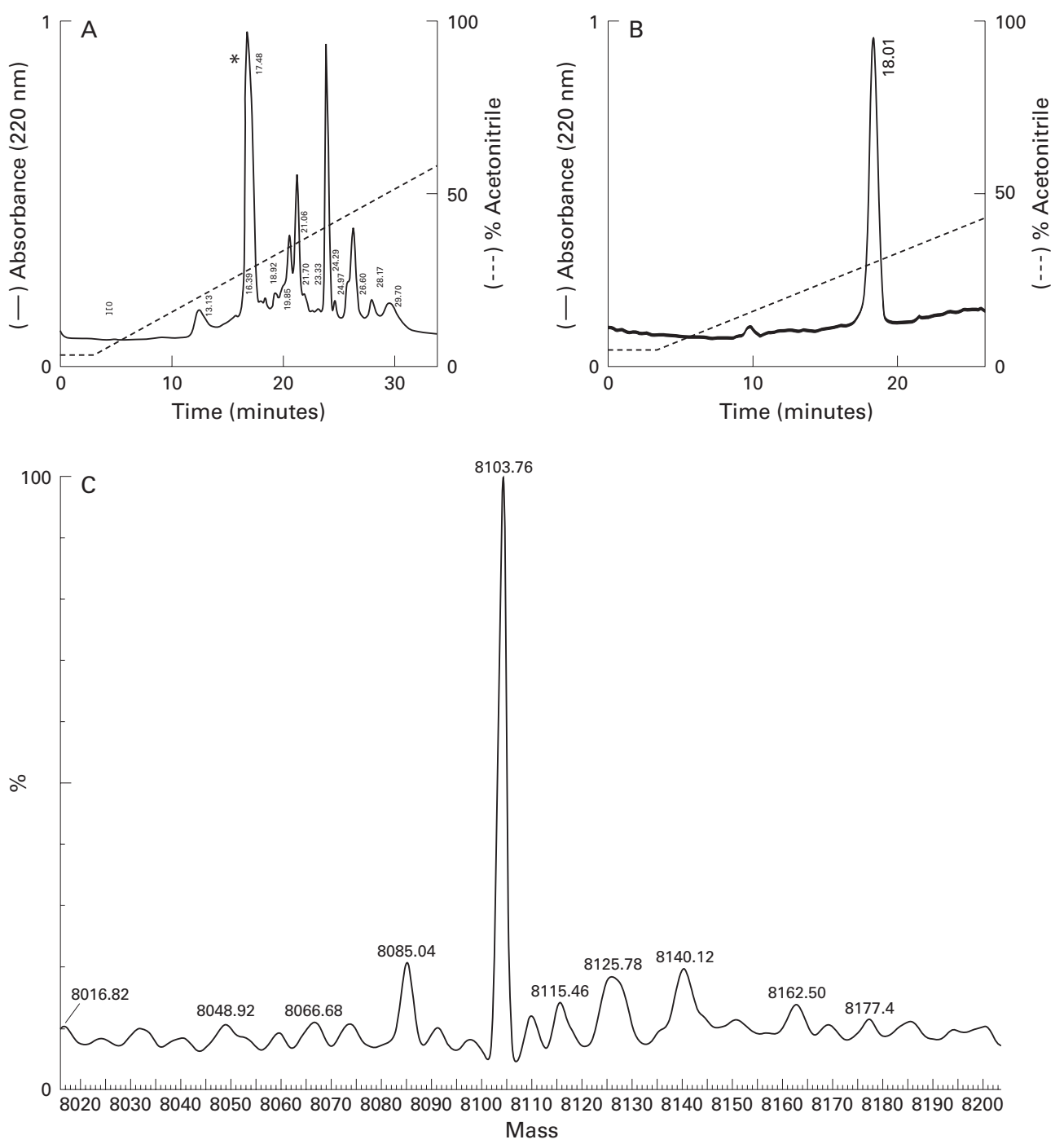

Figure 7 Purification and analysis of human defensin 5 (HD-5). (A) Pooled cation exchange fractions from the bracketed region in fig 6 were applied to a $C-18$ column. Individual eluted peaks were collected and screened for HD-5 immunoreactivity by immunoblotting. Only one fraction ( $\left.{ }^{*}\right)$ was reactive. This fraction was collected and reapplied to the column and the elution profile (B) suggested the presence of a single entity. $N$-terminal amino acid sequencing of this fraction gave the sequence ESLQERADEA, corresponding to the predicted $N$-terminus of the precursor form of $H D$-5 (fig 1). (C) Mass spectral analysis of purified HD-5. The sample (same as in B) was analysed in a standard Qtof 1 instrument and performed in positive ion mode. The figure was obtained following transformation of raw data using the MaxEnt 1 algorithm and shows that the major component is a peptide with a predicted mass of 8103.76 Da. The mass of pro-HD-5 predicted from the amino acid sequence (20-94, see fig 1) is $8103.83 \mathrm{Da}$.

that the major component was a peptide with a mass of 8103.76 (expected mass of the precursor form (amino acids 20-94) of HD-5 based on the amino acid sequence is $8103.83 \mathrm{Da}$ ). $\mathrm{N}$-terminal sequence analysis of this sample of purified HD-5 was found to be that of the predicted precursor form of the protein (amino acids 20-94, fig 1). These studies demonstrate that HD-5 is stored in the precursor form in normal terminal ileal Paneth cells. Interestingly, AU-PAGE and SDS-PAGE analyses of the same sample demonstrated the presence of more than one band but the same $N$-terminal sequence (that of the precursor form of $\mathrm{HD}-5$, amino acids 20-30) was present in all of them. The presence of more than one band with the same $N$-terminal amino acid sequence and a similar mass could be due to (i) unfolding and refolding of precursor form of HD-5 into different intramolecular disulphide linkages, (ii) variation in a single amino acid at the
C-terminus, or (iii) may represent an artefact of AU-PAGE.

In a recent study, Porter et al also identified the precursor form of HD-5 in terminal ileal mucosal extracts but found several truncated forms of the precursor protein and not the sequence of the predicted precursor form of HD- $5,{ }^{30}$ as in our study. As we consistently found only the $N$-terminal sequence of the predicted precursor form of HD-5, it is possible that the truncations of this protein demonstrated by Porter et al occurred during the isolation procedure. Our isolated precursor form of HD-5 did not demonstrate antimicrobial activity. The lack of antimicrobial activity of pro-HD-5 would also explain our previous unsuccessful attempts at its isolation from terminal ileal mucosal extracts using a sensitive antimicrobial assay. ${ }^{6}$ It is of interest that only the mature forms of cryptdins have been isolated from murine small intestinal Paneth 
A

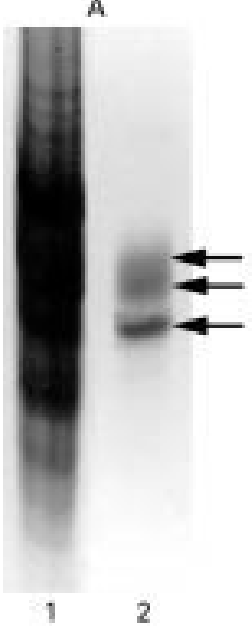

B

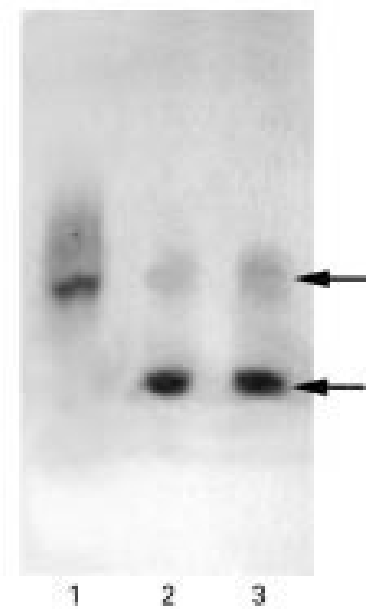

Figure 8 (A). AU-PAGE with Coomassie blue stain of crude acid extract of crypt epithelial cells (lane 1) and purified (by cation exchange chromatography and RP-HPLC; fig 7B) human defensin 5 (HD-5) (lane 2). $H D-5$ protein bands, as indicated by the arrows, were transferred to a PVDF membrane and sequenced directly. All three bands gave the identical $N$-terminal sequence of the precursor form of HD-5, ESLQERADEA. (B) $A U-P A G E$ western blot analysis of $H D-5$ secreted by terminal ileal crypts. Supernatants obtained from isolated ileal crypts cultured for one hour in the presence of $10 \mu \mathrm{g} / \mathrm{ml}$ lipopolysaccharide (lane 2) or $10^{-4} \mathrm{M}$ carbamylcholine chloride (lane 3) were subjected to AU-PAGE and transferred to a PVDF membrane and probed with anti-HD-5 antisera. Crude acid extract of crypt epithelial cells is included for comparison (lane 1). In addition to a band (upper arrow) corresponding to the precursor form of $H D-5$ (which is also seen in the crypt cell extract), an immunoreactive band of lower molecular weight (lower arrow) is present in the culture supernatants, corresponding to the truncated form of HD-5. Following RP-HPLC purification, $N$-terminal analysis of the truncated form of $H D-5$ revealed the sequence GEDNQDLAIS (amino acids 36-94).

cells. ${ }^{13-16}$ Recently it has been shown that the tissue metalloproteinase matrilysin is responsible for the processing of cryptdin precursors to their active mature forms within the murine Paneth cell. ${ }^{31}$ Human myeloid defensins are also stored in cytoplasmic granules in the mature form. ${ }^{32}$ Thus it is likely that processing of HD-5 in human Paneth cells (which do not express matrilysin; unpublished observations) differs from the processing of cryptdins in murine Paneth cells and that of human neutrophil defensins. Compared with myeloid defensins of humans and other species, the $N$-termini of cryptdins are extended by $4-6$ amino acid residues. ${ }^{13-16}$

The presence of only the precursor form of HD-5 in normal Paneth cells implies that processing to the mature form occurs during or after secretion into the lumen. To investigate this further, Paneth cells in isolated epithelial crypts were stimulated to secrete their granules using bacterial LPS and the cholinergic agonist carbachol. Western blot analyses following AU-PAGE and SDS-PAGE of supernatant samples consistently demonstrated the presence of a smaller form of HD-5, and following RP-HPLC purification and $N$-terminal sequencing this has been identified as an $N$-terminally truncated (by 16 amino acid residues; $N$-terminal sequence: GEDNQDLAIS) version of the precursor form of HD-5. In view of the lack of availability of adequate amounts, antimicrobial activity of this purified truncated from of HD-5 has not been determined. The truncated form of HD-5 identified in our studies ( $N$-terminal sequence: GEDNQDLAIS) has also been found to be one of the predominant forms of HD-5 present in the urine of ileal neobladder, ${ }^{30}$ implying that this step in the processing of the precursor form of HD- 5 does indeed occur in vivo.

Our results suggest that HD-5 is stored in ileal Paneth cells as the precursor form, and that its processing occurs during and/or after granule secretion. Further processing of the molecule may occur in the crypt lumen by endogenous proteases.

Dr Cunliffe was supported by Alimentary Pharmacology and Therapeutics Fellowship of the Digestive Disorders Foundation (UK). We thank Drs Gordon Kearney and Kirsten Hobby (of Micromass UK Ltd, Wythenshawe, Manchester) for assistance with the mass spectral analyses.

1 Simon G, Gorbach S. Normal alimentary tract flora. In: Blaser M, Smith $\mathrm{P}$, Ravdin J, eds. Infections of the gastrointestinal tract. New York: Raven Press, 1995:53-69.

2 Elson CO, Sartor RB, Tennyson GS, et al. Experimental models of inflammatory bowel disease. Gastroenterology 1995;109:1344-67.

3 Morales V, Snapper S, Blumberg R. Probing the gastrointestinal immune function using transgenic and knockout technology. Curr Opin Gastroenterol 1996;12:577-83.

4 Mahida YR, Rose F, Chan WC. Antimicrobial peptides in the gastrointestinal tract. Gut 1997;40:161-3.

5 Zasloff $M$. Antibiotic peptides as mediators of innate immunity. Curr Opin Immunol 1992;4:3-7.

nity. Curr Opin Immunol 1992;4:3-7.
6 Rose FR, Bailey K, Keyte JW, et al. Potential role of epithelial cell-derived histone $\mathrm{H} 1$ proteins in innate antimicrobial defense in the human gastrointestinal tract. Infect Immun defense in the humar

7 Rose FRAJ, Cunliffe RN, Mahida YR. Injured primary human colonic epithelial cells release specific antimicrobial activity while undergoing apoptosis. Gastroenterology 1999; 116: G3935.

8 Cunliffe RN, Rose FRAJ, James PD, et al. Expression of antimicrobial neutrophil defensin and lysozyme is induced in epithelial cells of active inflammatory bowel disease (IBD) mucosa. Gastroenterology 1999;116:G3936

9 Ouellette AJ. Paneth cells and innate immunity in the crypt microenvironment. Gastroenterology 1997;113:1779-84.

10 Mason DY, Taylor CR. The distribution of muramidase (lysozyme) in human tissues. 7 Clin Pathol 1975;28:124-32.

11 Harwig SS, Tn I murine murine in

12 Nevalainen TJ, Gronroos JM, Kallajoki M. Expression of group II phospholipase A2 in the human gastrointestinal group II phospholipase A2 in the
tract. Lab Invest 1995;72:201-8.

13 Ouellette AJ, Miller SI, Henschen AH, et al. Purification and primary structure of murine cryptdin-1, a Paneth cell defensin. FEBS Lett 1992;304:146-8.

14 Selsted ME, Miller SI, Henschen AH, et al. Enteric defensins: antibiotic peptide components of intestinal host defense. F Cell Biol 1992;118:929-36.

15 Ouellette AJ, Hsieh MM, Nosek MT, et al. Mouse Paneth cell defensins: primary structures and antibacterial activities of numerous cryptdin isoforms. Infect Immun 1994;62: $5040-7$

16 Eisenhauer PB, Harwig SS, Lehrer RI. Cryptdins: antimicrobial defensins of the murine small intestine. Infect Immun 1992;60:3556-65.

17 Lencer WI, Cheung G, Strohmeier GR, et al. Induction of epithelial chloride secretion by channel-forming cryptdins 2 and 3. Proc Natl Acad Sci USA 1997;94:8585-9.

18 Jones DE, Bevins CL. Paneth cells of the human small intestine express an antimicrobial peptide gene. 7 Biol intestine express an antimict

19 Jones DE, Bevins CL. Defensin-6 mRNA in human Paneth cells: implications for antimicrobial peptides in host defense of the human bowel. FEBS Lett 1993;315:187-92.

20 Porter EM, Liu L, Oren A, et al. Localization of human intestinal defensin 5 in Paneth cell granules. Infect Immun 1997;65:2389-95.

21 Porter EM, van Dam E, Valore EV, et al. Broad-spectrum antimicrobial activity of human intestinal defensin 5. Infect Immun 1997;65:2396-401.

22 Podolsky DK, Lynch-Devaney K, Stow JL, et al. Identification of human intestinal trefoil factor. Goblet cell-specific expression of a peptide targeted for apical secretion. 7 Biol Chem 1993;268:6694-702.

23 Mahida YR, Galvin AM, Gray T, et al. Migration of human intestinal lamina propria lymphocytes, macrophages and eosinophils following the loss of surface epithelial cells. Clin Exp Immunol 1997;109:377-86.

24 Fields PI, Groisman EA, Heffron F. A Salmonella locus that controls resistance to microbicidal proteins from phagocytic cells. Science 1989;243(4894 Pt 1):1059-62. 
25 Troughton WD, Trier JS. Paneth and goblet cell renewal in mouse duodenal crypts. F Cell Biol 1969;41:251-68.

26 Calvert R, Bordeleuu G, Grondin G, et al. On the presence of intermediate cells in the small intestine. Anat Rec 1988; 220:291-5

27 Geller SA, Thung SN. Morphologic unity of Paneth cells. Arch Pathol Lab Med 1983;107:476-9.

28 Quayle AJ, Porter EM, Nussbaum AA, et al. Gene expression, immunolocalization, and secretion of human defensin-5 in human female reproductive tract. Am f Pathol 1998;152:1247-58.

29 Diamond G, Bevins CL. Beta-defensins: endogenous antibiotics of the innate host defense response. Clin Immu-

nol Immunopathol 1998;88:221-5.
30 Porter EM, Poles MA, Lee JS, et al. Isolation of human Porter EM, Poles MA, Lee JS, et al. Isolation of human
intestinal defensins from ileal neobladder urine. FEBS Lett intestinal defensins

31 Wilson CL, Ouellette AJ, Satchell DP, et al. Regulation of intestinal alpha-defensin activation by the metalloproteinase matrilysin in innate host defense. Science 1999;286: 113-7.

32 Ganz T, Selsted ME, Szklarek D, et al. Defensins. Natural peptide antibiotics of human neutrophils. 7 Clin Invest 1985;76:1427-35. 